\title{
A PRELIMINARY NOTE ON THE EMPLOY- MENT OF CERTAIN CONSTITUENTS OF THE GONOCOCCUS IN TREATMENT AND OF OTHER CONSTITUENTS IN TESTS OF CURE*
}

By MAJOR E. C. LAMBKIN, D.S.O., M.B., R.A.M.C.

THE work which I am about to attempt to describe has been carried out in the Venereal Division of the Royal Herbert Hospital, Woolwich, which is the present representative of the late Military Hospital, Rochester Row.

The particular line of treatment for gonorrhœa which we have been investigating is the direct result of researches by Captain Lyn Dimond, R.A.M.C., in the chemistry and biology of the gonococcus, at which he has been working for the last three years. Captain W. J. Robertson, R.A.M.C., is mainly responsible for the clinical application of it.

Speaking generally, the objects of this work are :-

(I) To test the value in treatment of certain fractions or products of metabolism of the gonococcus employed locally and intradermally.

(2) Similarly to test the value of these fractions or products of metabolism as provocative agents in deciding the question of cure.

(3) Having determined-

(a) The conditions under which the gonococcus elaborates antigenic or immunising properties, and

(b) Those under which it elaborates only toxic properties and develops resistance forms :

To test the value of chemical treatment designed to put the gonococci in the patient's tissues under the least favourable conditions of vitality.

By special cultural methods, it was found that when gonococci are grown on a medium which, broadly speak-

- Based upon an address delivered before the Medical Society for the Study of Venereal Diseases, November 26 th, 1926. 


\section{BRITISH JOURNAL OF VENEREAL DISEASES}

ing, is rich in animal nucleo-protein, approximately onethird of the isolated strains develop polar bodies (Babes' bodies), which can be demonstrated histologically by staining methods identical with those employed in the demonstration of the polar bodies in $B$. diphtheria. These polar bodies are composed of alpha-nucleo-protein and beta-nucleo-histone.

They are only loosely attached to the bodies of the gonococci, and can be separated easily from these by washing off the culture with 2 per cent. saline and either fractionally centrifugalising the emulsion or, by means of gravity, the polar bodies being found in the supernatant fluid. Since this dissolves the beta-nucleo-histone, the solid elements in the supernatant fluid are the alphanucleo-protein fraction of the polar bodies.

Clinical experiments have shown that both the alphanucleo-protein and the beta-nucleo-histone are antigenic (the beta-nucleo-histone more so than the alpha-nucleoprotein), and relatively non-toxic, so that they appear eminently suitable for use in the vaccine treatment of gonorrhœa.

For intradermal injection we employ them in a strength which represents the product of I,OoO million gonococci per cubic centimetre in 2 per cent. salt.

For intra-urethral injection we standardise them in 2 per cent. saline, so that the nucleo-protein of I0o,000 million organisms is contained in $\mathrm{I}_{5}$ c.c. We prefer to employ much larger doses than this, and better results are obtained with three and four-fold this dosage; but such large doses are limited by the corresponding large amount of antigen which must necessarily be grown. To this I5 c.c., representing, say, the product of I00,000 million gonococci, is added I per cent. of sodium taurocholate, and the reaction brought to a $p \mathrm{H}$ of $7 \cdot 2$. Mucin (either commercial or from umbilical cords) is then added to Ioo c.c. The dose is 20 c.c. for intra-urethral injection, or the product of 20,000 million gonococci.

At first we reduced the strength of the suspending and dissolving saline to I per cent. by dilution with normal saline, but have more recently employed the alphanucleo-protein precipitate and beta-nucleo-histone solution in the original 2 per cent. saline. This does not cause tissue reaction, and has the advantage that it maintains the alpha-nucleo-protein fraction in the solid form and 
prevents the formation of a small amount of toxin from the dissolved alpha-nucleo-protein, thus permitting the employment of the product in larger doses.

So far as our work has gone it has shown that the antigenic properties of a gonococcal vaccine are practically all contained in the alpha-nucleo-protein and beta-nucleohistone components of the polar bodies, and that the remainder of the gonococcal body is undesirable for use in immunisation.

It is interesting, in view of the observation of various workers that ordinary gonococcal vaccines seem to vary in antigenic value, that only one-third of the strains we have isolated have produced the antigenic polar bodies.

We have so far employed our vaccine in the following way:-

The patient is first irrigated thoroughly with a weak solution of potassium permanganate (strength $I$ in 20,000 ), and then 20 c.c. of the vaccine in mucin and taurocholate of soda are injected into the urethra, steps being taken to secure that the vaccine is retained within the urethra for as many hours (three or more) as possible.

At the same time the patient receives an intradermal injection of $\frac{1}{5}$ c.c. of the same vaccine, corresponding to 200 millions of the original culture.

The general treatment of the patient is based on the result of our observations of the conditions which are favourable to the production of the antigenic polar bodies and those favourable to the production of toxin.

The discussion of these observations need not be detailed here, but generally they showed that the most favourable reaction of the tissues from the point of view of resulting gonococcal invasion is that when the $p \mathrm{H}$ value of the urine is within the limits of $7 \cdot 2$ and $7 \cdot 4$.

When the urine is on the acid side of this figure the many gonococci in the secretion are in tetrad formation protected by great amounts of sclero-protein, and, as such, in resistance formation; on the other hand, when it is on the alkaline side, there is considerable autolysis with liberation of irritative endotoxin.

After testing a number of alkalies to obtain the optimum reaction of the urine, we found that di-sodiummono-hydrogen phosphate appeared to serve the purpose most suitably.

The administration of this alkali is controlled by a 


\section{BRITISH JOURNAL OF VENEREAL DISEASES}

daily titration of the patient's urine. After the first injections daily observations are made of the urethral discharge, and the intra-urethral and intra-dermal injections are repeated according to various circumstances.

In the case of the intra-dermal injection usually there is a ten-day interval between injections.

In the case of intra-urethral injection it is usually repeated next day and ensuing days should the urethral discharge still show gonococci. Latterly our practice has been to raise the initial dosage of this injection and to administer at less frequent intervals.

Again, the arrival of a patient's urine to the optimum $p \mathrm{H}$ value from being acid when he received his initial injection is another indication for a repeated intraurethral injection. A patient whose condition has been deliberately provoked into activity by an endotoxin injection receives an intra-urethral non-toxic injection as soon as this condition is recognised. Lastly, in the subacute and chronic stages a positive gonococcal discharge is an indication for a non-toxic injection.

We do not hold that the dosage and intervals which we have mentioned are the best. Indeed, we think that possibly we may be able to administer much larger doses now that by employing 2 per cent. saline throughout we have practically eliminated the formation of toxin in the vaccine. We have frequently employed such doses as correspond to 80,000 million of the organism with promising results and no sign of provocation, but we have so far been influenced by the difficulty of producing sufficiently large amounts of the vaccine to permit of our using it in greater strength or more frequently.

The results of the treatment we have carried out on these lines have been various, and we cannot do better than relate the course of events in favourable and in unfavourable cases. In cases which progress favourably the usual course is as follows. After an injection intraurethrally of 20 c.c. of the vaccine the discharge rapidly diminishes, with a corresponding decrease in the numbers of gonococci in films. The case goes on to cure, and subsequent instillations of endotoxin fail to cause relapse.

So far as we have been able to determine this happy sequence of events is the common course taken by two classes of case :-

(I) The early anterior urethritis. 
(2) The cases with previous history of gonorrhœa but who have seen no signs for, sometimes, years, and who have recently been exposed to infection, in fact, as far as one can determine, a reinfection.

Charts showing types of cases are given on pp. 38-45.

Our theory as to the probable course of events is that-

(I) In an early case, it would appear that the gonococci are caught before they have had time to bury themselves deeply in follicles and that the local and general immunity response is great enough to protect the urethra from spread of infection. The examination of the urethra later fails to reach the local foci or infiltrations and there is no involvement of the posterior urethra.

(2) In the reinfected case-here one assumes that the old urethritis denuded the urethra of most of its luxuriant columnar epithelium, which is such a good medium for gonococcal growth, and the remaining portions invaded by the second attack are more easily reached and reacted upon by the local vaccine, and rapid resolution takes place; in short the gonococcus is more vulnerable in such cases, although this idea is contrary to the opinion of such an expert as Luys. One must, however, not neglect the fact that in these cases there may be such a factor as some degree of acquired general immunity.

In cases which have not pursued such a favourable course it is very difficult to find some common factor, and it is more convenient to discuss the types that have been noted clinically.

The usual course is an initial amelioration of all signs and symptoms as in the favourable type of case. This naturally leads to great hopes.

However, somewhere about the tenth to fourteenth day a discharge again appears, in about half the cases showing scanty gonococci. In the majority of cases this is never more than a subacute phenomenon, and the case soon settles down and proceeds as in the more favourable type.

In others this exacerbation may be more prolonged, and symptoms arise which vary from a mild degree of irritation of the posterior urethra to a severe frank posterior urethritis. Between the two extremes one will find all degrees of severity.

The time of resolution varies according to the severity; 


\section{BRITISH JOURNAL OF VENEREAL DISEASES}

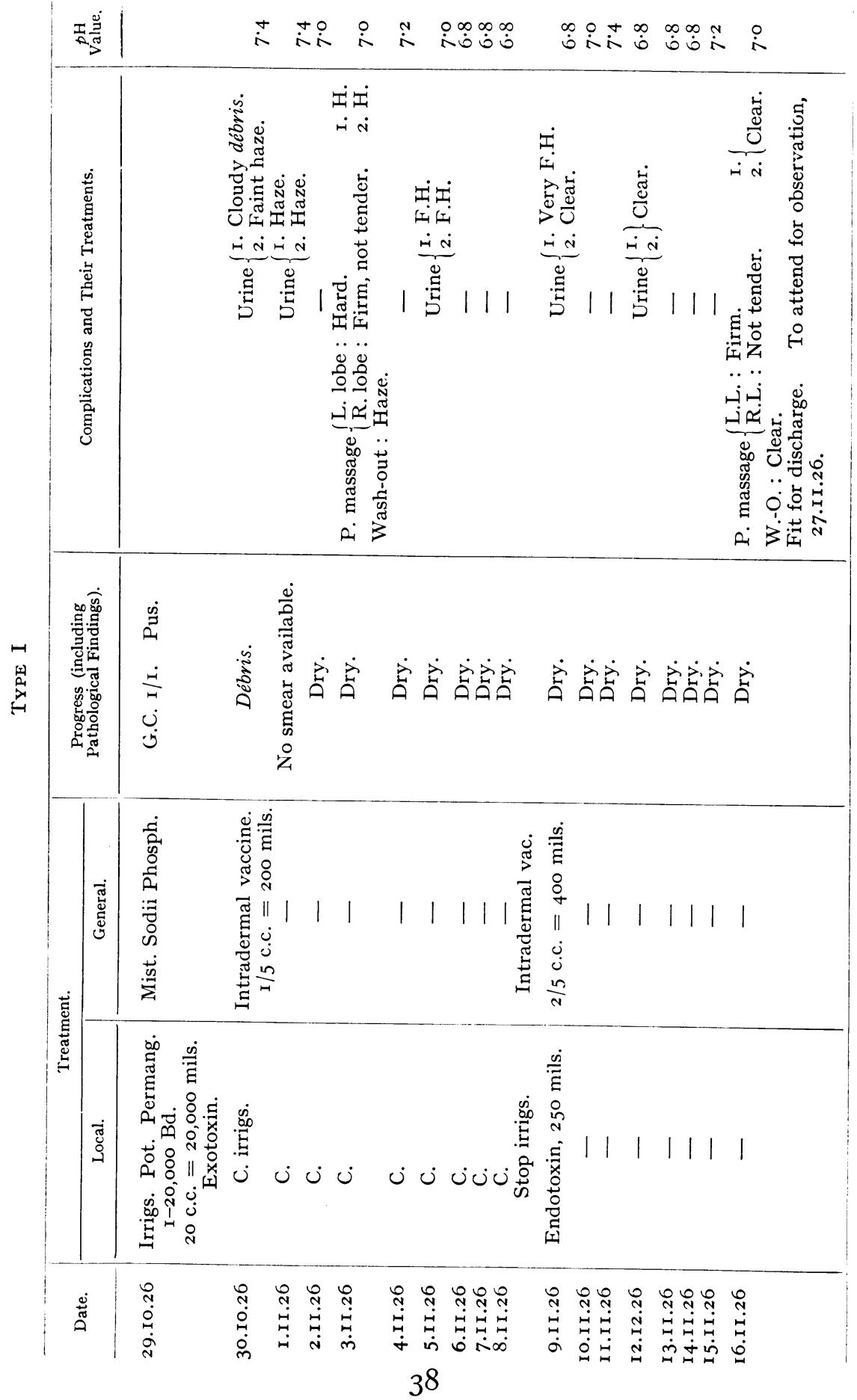


CONSTITUENTS OF THE GONOCOCCUS, ETC.

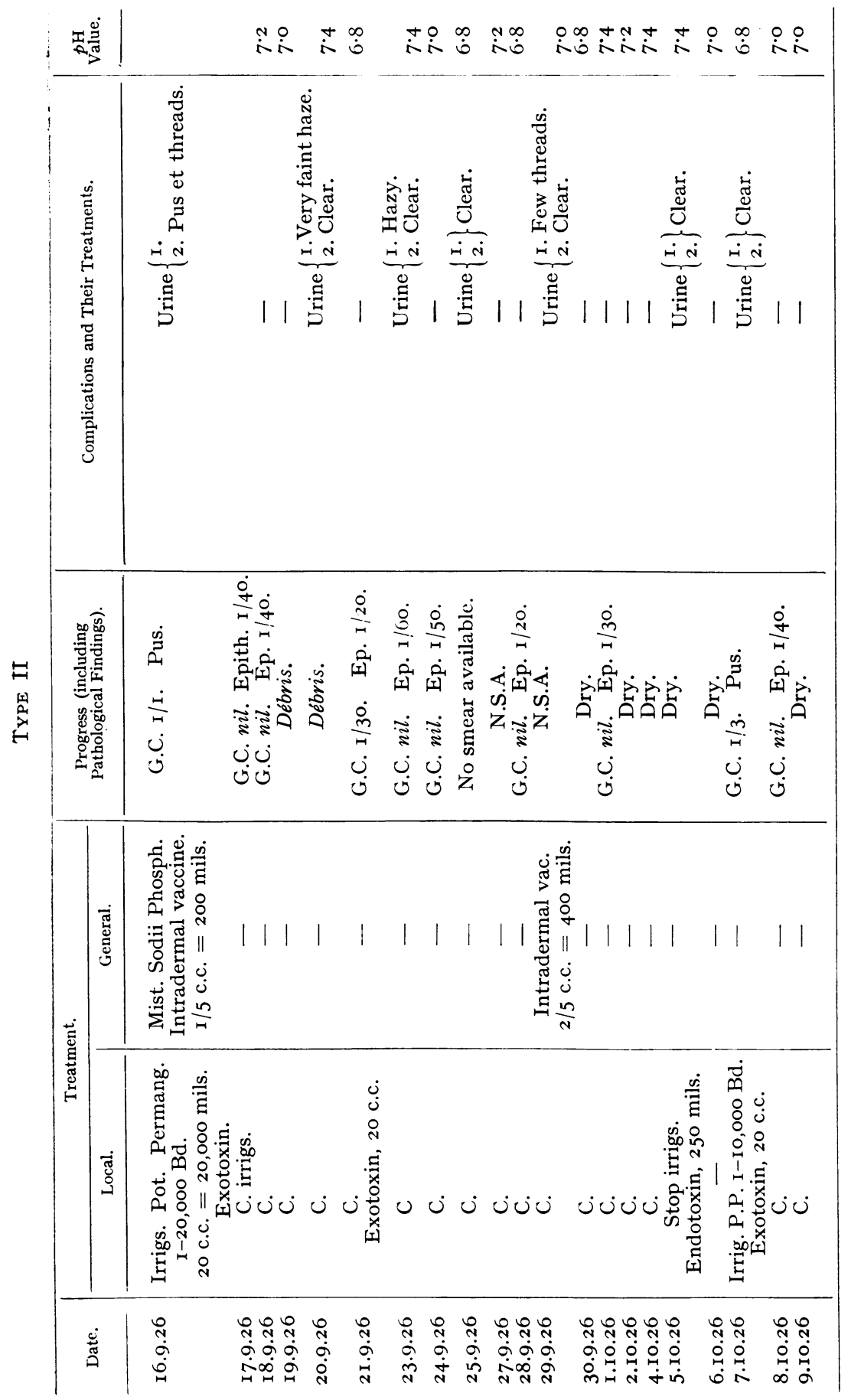


BRITISH JOURNAL OF VENEREAL DISEASES

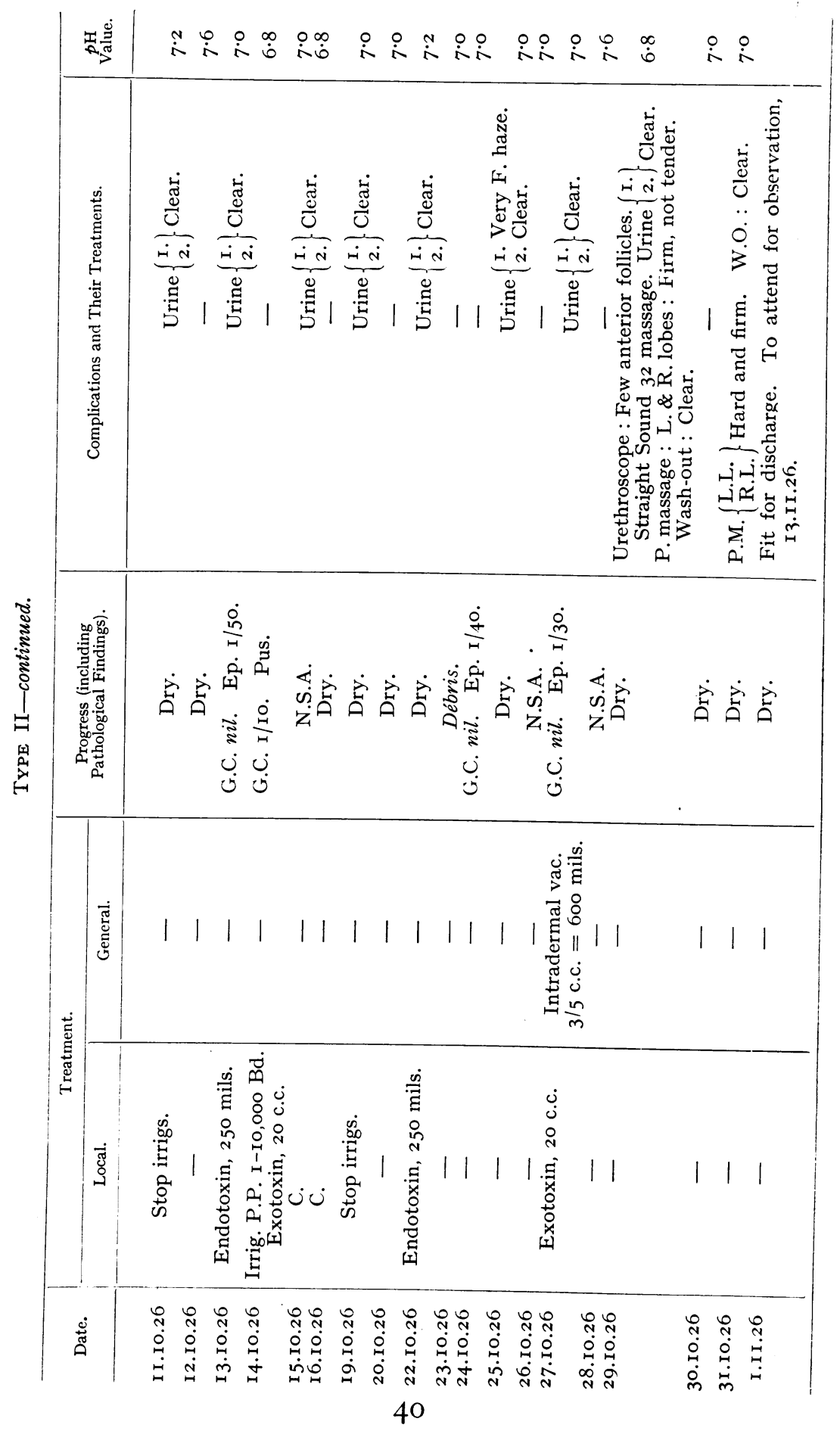

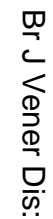

जo 
I CONSTITUENTS OF THE GONOCOCCUS, ETC.

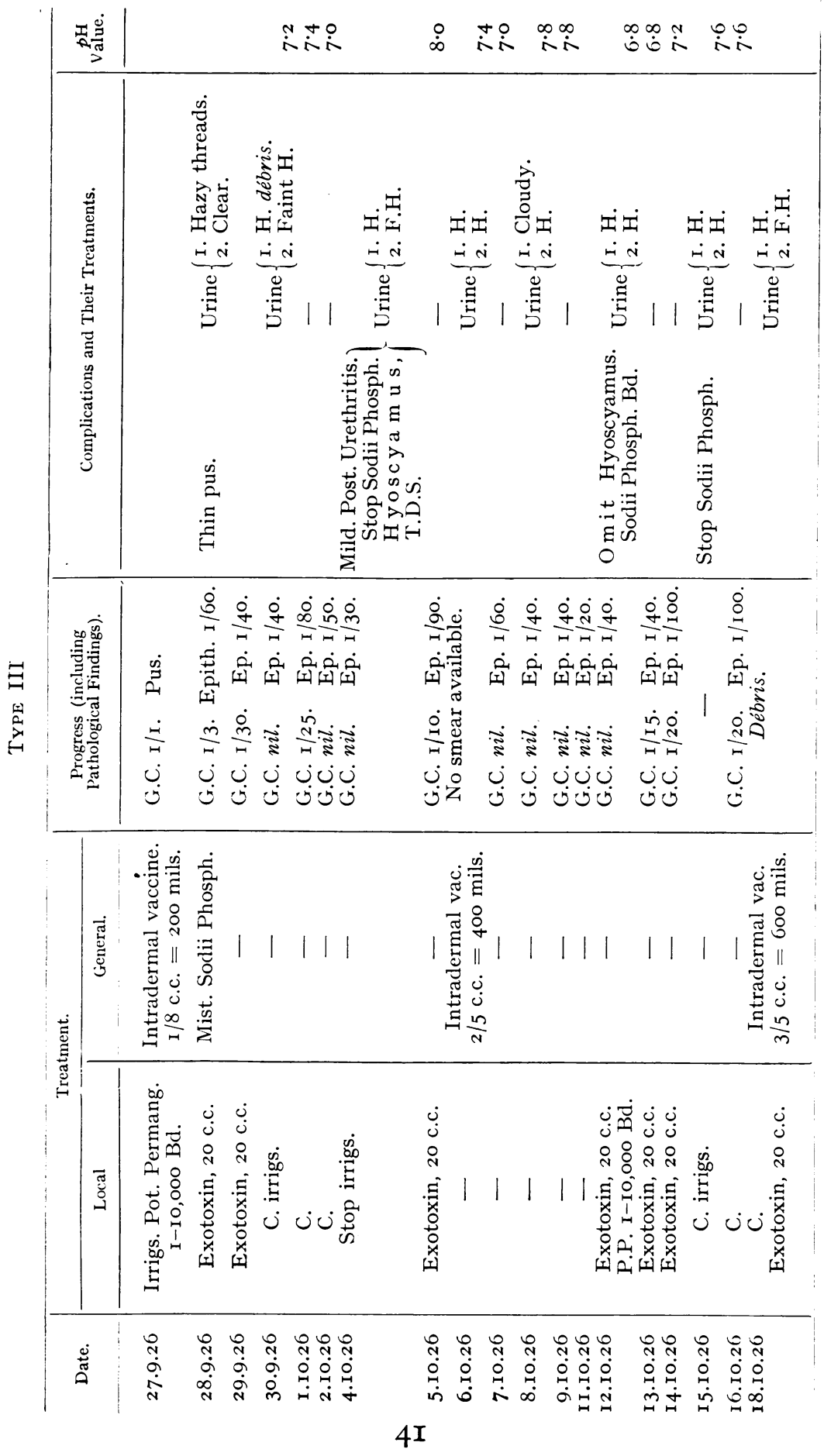

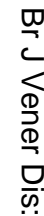

ज) 
BRITISH JOURNAL OF VENEREAL DISEASES

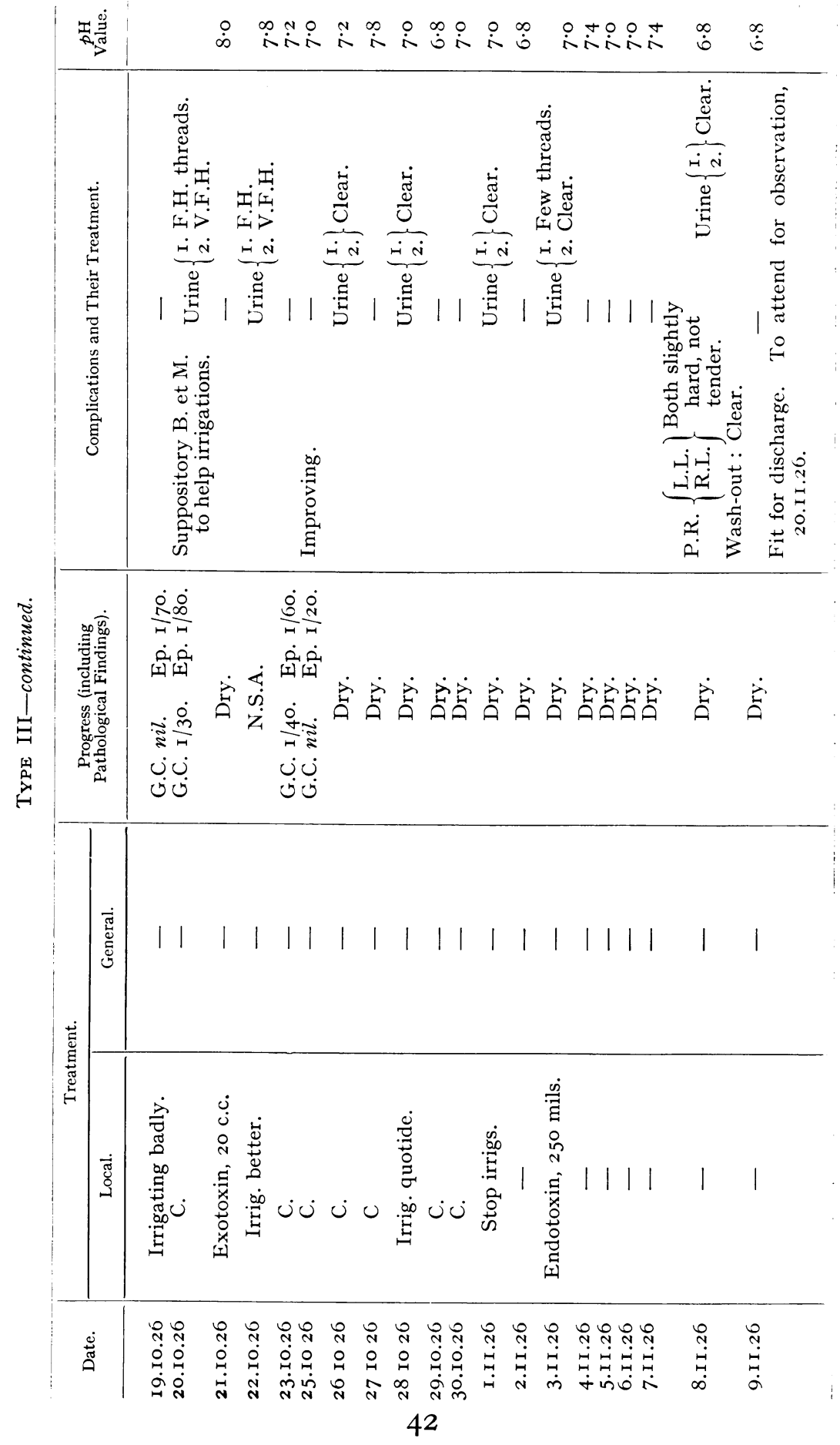


CONSTITUENTS OF THE GONOCOCCUS, ETC.

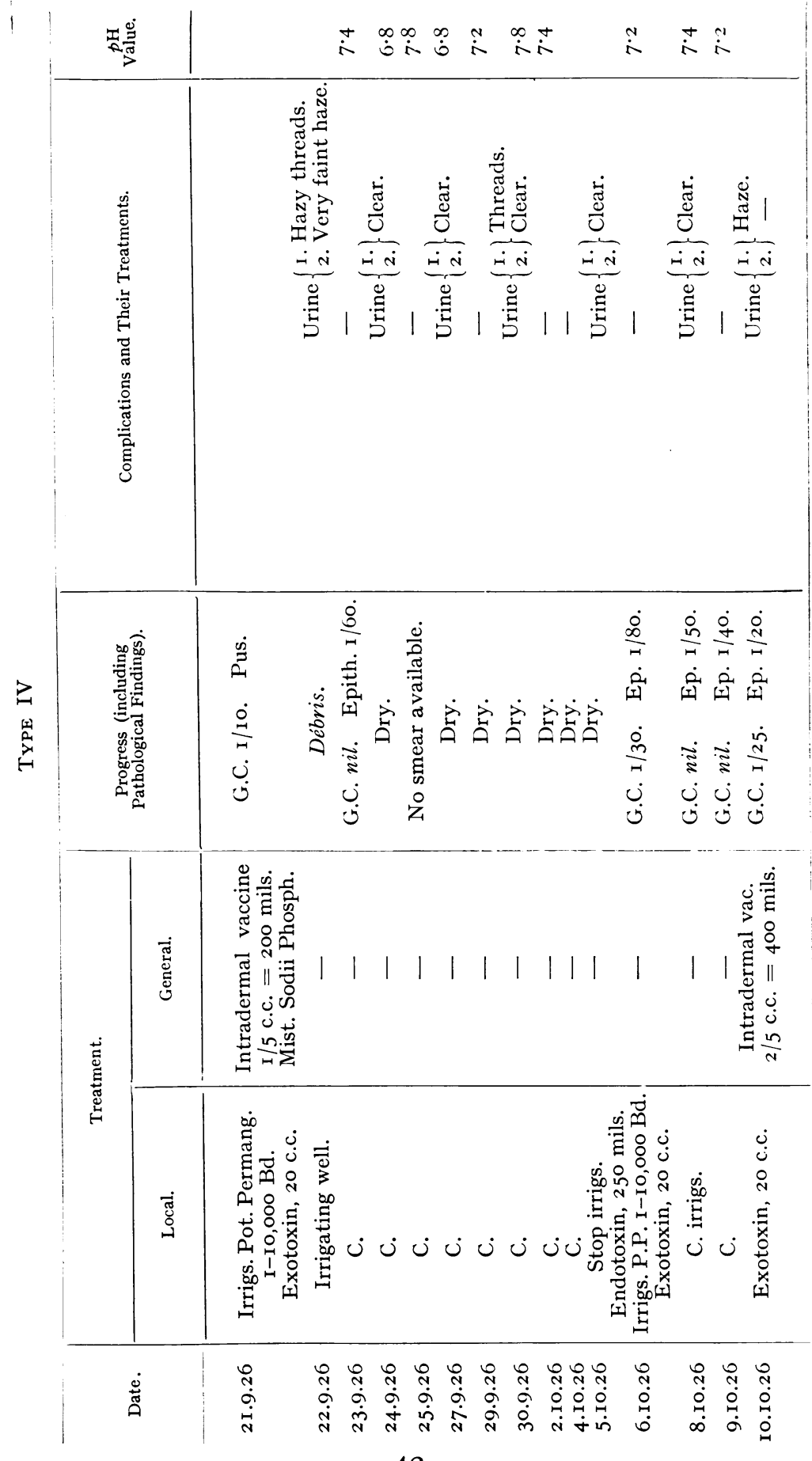


BRITISH JOURNAL OF VENEREAL DISEASES

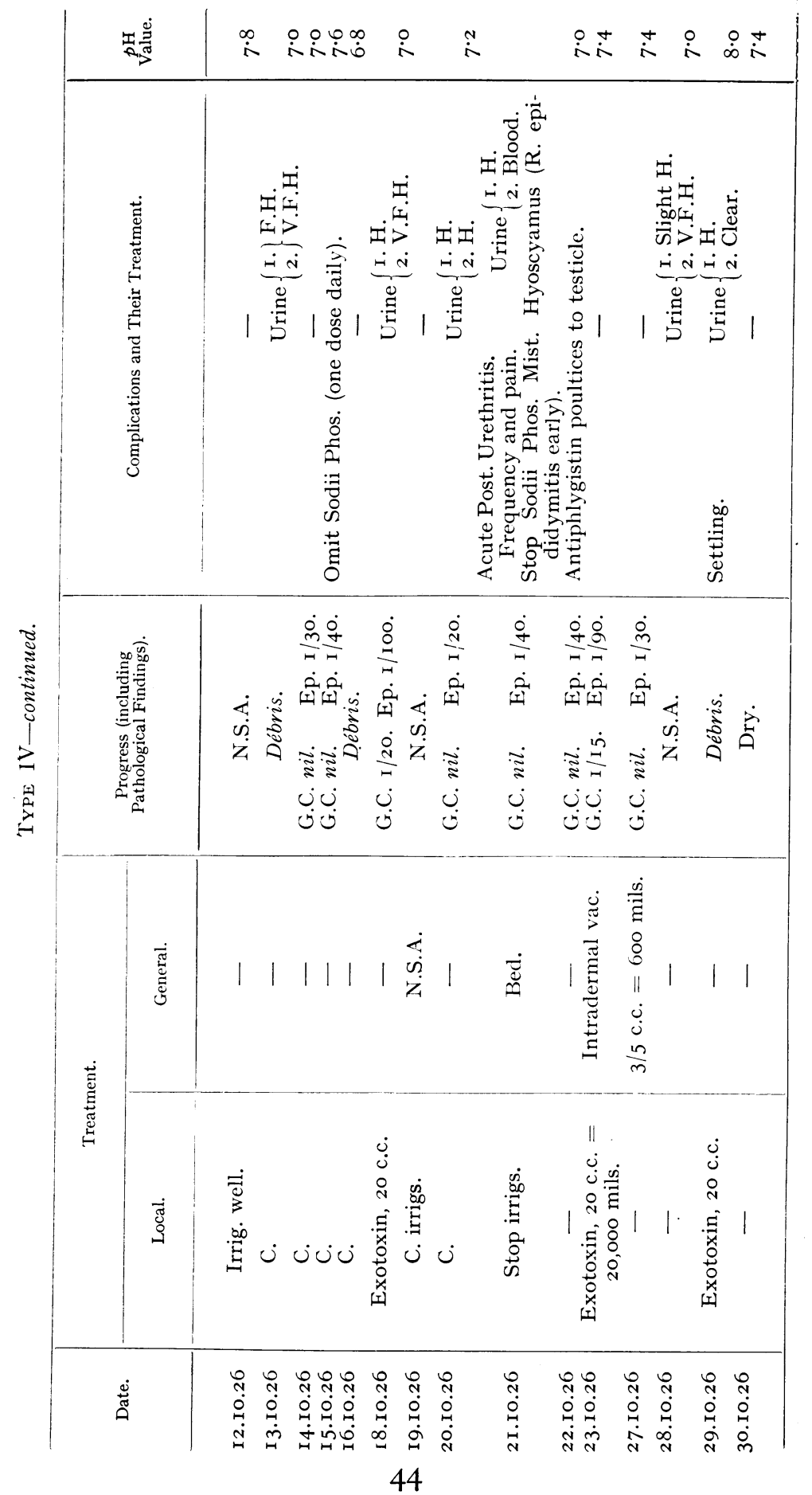

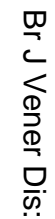

ज) 
C.ONSTITUENTS OF THE GONOCOCCUS, ETC.

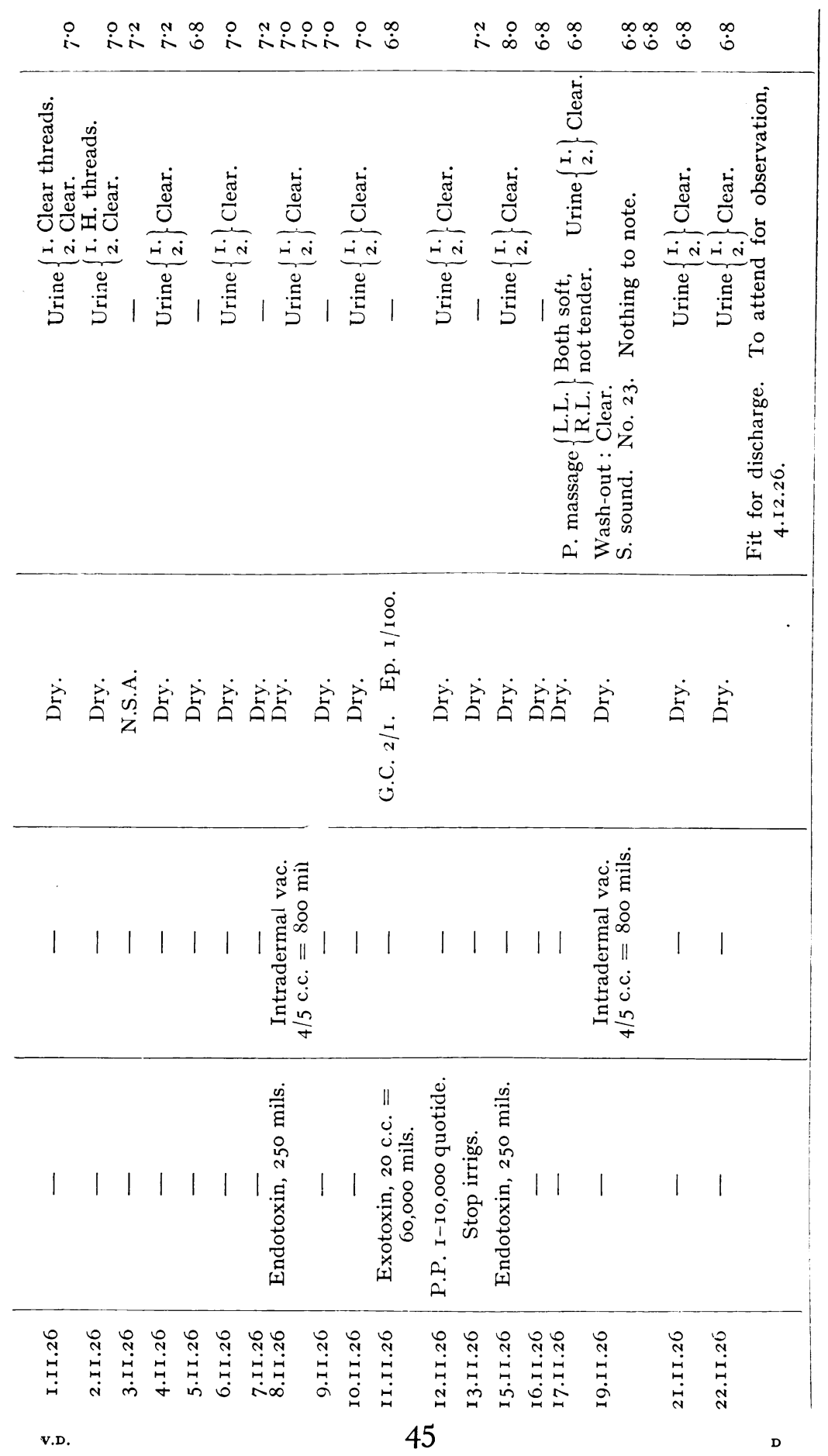

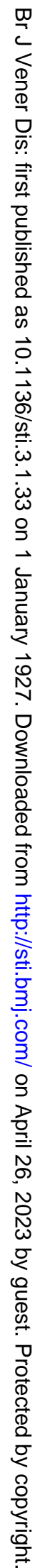




\section{BRITISH JOURNAL OF VENEREAL DISEASES}

a feature common to all is the difficulty of finding gonococci in urethral smears, and having found them, the impossibility of growing them.

We have tried to interpret the course of events as follows :-

(I) In the milder cases the infection remains anterior, and most of the gonococci are dealt with by the initial vaccine application, but some penetrate follicles and remain there untouched by the superficial immunising process ; they, however, get into the urethra again about the tenth to fourteenth day, and it may depend on the condition of the urethra as to whether or not they make subsequent headway there.

(2) Perhaps a condition of inflammatory œedema may exist at the time of the initial treatment and the vaccine never gets into contact with the process except in isolated less inflamed parts. In such cases there always remain resistant foci constituting a chronic folliculitis, but infiltrations are never encountered to such degrees as one is accustomed to see in these cases.

The same condition may be present behind the compressor muscle with the involvement of the accessory glands. It is in such cases that one has to rely on the intradermal route of administration for the stimulation of the necessary antigenic substances.

I am conscious that this is a very sketchy synopsis of cases. I feel the results of treatment of a few hundred cases, when dealing with a disease such as this, to be of little value when trying to draw conclusions. The burning question from the Army point of view is " days in hospital." So far I am afraid this side of the question has not influenced me; we have been trying to learn and find out optimum conditions and dosage. So that I cannot tell you how rapidly we have cured cases as a whole. The points that have impressed us are that:-

Speaking broadly, we are conscious of a general and sustained improvement in the cases as a whole.

In a large clinic such as ours the daily observations of urethral smears point to an enormous decrease in the number showing gonococci, the urethral discharge quickly becomes scanty, and symptoms ameliorate with almost astonishing rapidity in favourable cases.

The incidence of complications such as epididymitis, severer forms of prostatitis, arthritis, etc., has fallen, and 
the fact that cases so treated have not relapsed has been a most encouraging sign and given us hope that we might be on the right lines towards initiating a line of investigation which would cure what has been an incurable disease.

Certain lessons seem to emanate from our results :-

(I) The importance of stabilising the patient's reaction by daily observation of his urine $p \mathrm{H}$. Speaking generally, a constant acid urine indicates the optimum condition for resistance forms of gonococci and manufactures deep-seated encysted foci, be they in follicles or in any of the urethral annexa. On the other hand, a continuous highly alkaline urine of $p \mathrm{H} 8$ in many cases will produce an acute posterior urethritis and all its potentialities.

(2) The washing of the culture must be carried out with 2 per cent. saline.

At an earlier stage the use of serum for washing off the culture and suspension of the nucleo-proteins had the disadvantage of liberating toxin from the protein element of the nucleo-protein and the result was irritative. The use of serum in this connection had the further disadvantage that it was difficult to sustain the supply of the large quantities required for the purpose.

It will be realised from what we have said that we are far from having elaborated a perfect line of treatment, but we think that a number of our results have been sufficiently good to indicate that in immunisation by the alpha-nucleo-protein and the beta-nucleo-histone components alone, eliminating the toxic element, we have at principle of treatment which is worthy of further investigation.

We have ventured to publish the method at this comparatively early stage because the principle we have expounded seems to open out a new line of attack on gonorrhœa and because we hope that others may perhaps be inclined to pursue the investigation on similar principles, so that we may attain the long desired control of gonococcal infection more quickly than would be possible by our working alone with our somewhat limited facilities and material.

In this connection it is necessary to say here, that having less of the vaccine than we can use ourselves, we have none to spare. Workers who wish to try this treatment on the lines we have indicated must, therefore, make their own arrangements for the manufacture of 


\section{BRITISH JOURNAL OF VENEREAL DISEASES}

the vaccine, and we can only offer to supply such details as will enable them to repeat our work.

A special caution is necessary here.

Unless very careful precautions are taken, it is easy to make a vaccine which is not only poor in antigen but highly toxic and irritative, so that we would ask workers to carefully identify their samples.

Otherwise we fear that the method or perhaps the principle of our treatment may suffer undeserved discredit.

\section{The Use of Endotoxin as a Test of Cure}

For the preparation of endotoxin we have used strains which do not throw out the polar bodies employed in our vaccine. These strains comprise 66 per cent. of those we have isolated.

They are grown for ten days on an acid autolysate of ox heart.

The culture is washed off, and after being repeatedly frozen and thawed yields a product of albumoses in a blue opalescent solution.

It is made up to a strength representing the product of 250 million gonococci in I c.c., the diluent being chemically prepared colloidal silver.

The effect of an intra-urethral injection of this preparation in a quiescent gonococcus carrier is to provoke his disease into temporary activity with the appearance of gonococci in the urethral discharge.

This product we believe to contain the true toxin elements of the gonococcus.

We have employed it as a routine for nearly four years now as a test of cure before a patient is discharged from hospital. The provocative effect may be observed as early as twelve hours or may be delayed for several days. We have seen it delayed for as long as a week, and to legislate for these delayed reactions, we observe the patient for seven days after an injection of endotoxin before we pronounce him free from active signs.

The great majority of cases when once provoked by endotoxin seem to do much better after the "flair up " of their disease, at least it is rarely necessary to have to give three or four injections of endotoxin at the end of observation periods for test of cure. This fact has induced us recently to administer endotoxin earlier in 
the course of treatment, not so much as a test of cure but to provoke into activity and the emptying of their contents the possible deeper submucous foci, which might in the ordinary course have become sealed up or encysted, only to break down and empty their infective contents into the urethra later, and thus prolong the proceedings.

In certain types of cases this early provocation with endotoxin has been successful, but as routine it is a risky business under three weeks from the onset of the attack, and we have observed it plunge a case that was doing very well under our routine treatment into a prolonged "negative phase" and in a few instances a posterior urethritis.

This condition of " negative phase" had never been observed after endotoxin injections employed as a test of cure, but it is a likely possibility if an attempt is made to "hurry things up a bit" without carefully selecting suitable cases.

After any observed provocation following an endotoxin injection the special intra-urethral vaccine is at once injected.

There has been much previous work by various observers which seemed to point to the nucleo-proteins as containing or being in some way connected with antigen or the immunising complexes. For instance, Sidney Rowlands' plague vaccine consisted of nucleo-proteins extracted from the $B$. pestis.

Captain Dimond, at Rochester Row, in I922, extracted the nucleo-protein from a considerable bulk of gonococcus culture and found it to be very highly antigenic both by its action when injected into the urethra in acute gonorrhœa and also when injected intra-peritoneally into mice four days before 2 M.L.D. of living gonococcus culture were administered with no obvious action upon the health of the animal. It also caused the production of amboceptor in high titre when injected into rabbits.

In the case of other organisms a multitude of observers, and in the case of the gonococcus Vannod, of Berne, all gave satisfactory experimental evidence of high antigenic value of the germ nucleo-protein fraction.

Unfortunately, all the samples of nucleo-protein so obtained were toxic, due to this admixture of the nucleoprotein with endotoxin, and the small amount (some 5 


\section{BRITISH JOURNAL OF VENEREAL DISEASES}

to Io per cent. of the dry weight of the organism) made its isolation in large bulk for therapeutic purposes impracticable. Later, at Woolwich, it was found that the gonococcus when grown on media containing animal nucleic acid and protamines or histones developed an extraordinary increased amount of nucleo-protein in approximately 33 per cent. of all strains examined up to date, this increased nucleo-protein content being evidenced, as referred to above, in the presence of polar bodies. As the result of these "polar bodies" the nucleo-protein content of the cultures was found to be from 50 to 55 per cent. of the dried weight of the organism instead of the 5 to ro per cent. found in cultures grown on ordinary media. In order to further stimulate this nucleo-protein content all the animal and plant nucleic acids available were tried, and as a result it was found possible to stimulate roo per cent. production of polar bodies in the case of the gonococcus with animal nucleic acid alone.

Again, a good deal of attention has lately been drawn to the soluble products of bacterial metabolism in normal saline as a result of Horder and Ferry's investigations. Knowing the small nucleo-protein content of practically all organisms and the peculiar solubility reactions of nucleo-proteins, it seems likely that the antigenic value of such washes deprived of the bodies of the organisms is due to their content of dissolved alpha-nucleo-protein.

The following observations seem to indicate that any antigenic action possessed by these germ nucleo-proteins is more an expression of simple nucleic acid chemistry than of specific origin. Wooldridge, for example, was able to produce immunity to anthrax in rabbits by means of thymus alpha-nucleo-protein. We have also noted that mice that have received $20 \mathrm{mgm}$. of dried rabbit thymus intraperitoneally, when inoculated four days later with 2 M.L.D. of living gonococcus, survive without illness, as they do when the specific gonococci nucleoproteins are used.

Lastly, when considering the remarkable antigenic powers of Calmette's tuberculin which are reported, it seems likely, if our own results turn out to be substantiated, that these reported results are true and that he is employing a vaccine in which he has stimulated nucleoprotein production. The media which he employs for 
growing the tubercle bacillus for the preparation of tuberculin is one which contains ox bile, and it is possible that its antigenic powers are due to the fact that ox bile has the true mucin found in human bile replaced by a nucleo-protein and that a considerable percentage of this nucleo-protein is present.

As regards media employed in these various preparations. I have mercifully omitted details as to preparation of quantities for two reasons :-

(I) It would be intolerable to listen to, and-

(2) Only this week further slight modifications are being tried. These details are best left for publication.

Speaking very roughly, there are three media in use :-

\section{(I) Isolation Media}

There is little interest here. Most workers have their special media, ours is an alkaline autolysate of ox heart. But I do not think that any special media are required for isolation, the main thing is to induce gonococci to grow when you require them in primary culture.

Having got a strain growing, one next proceeds to try and isolate a polar body producing strain. To do this it is necessary to transfer to-

\section{Media No. 2}

Which are the ordinary isolation media enriched either by :-

(I) Whole thymus gland or the extracted nucleoproteins, viz.: alpha-nucleo-protein and beta-nucleohistone ; or-

(2) Ripe cod sperm, which consists chiefly of histone nucleate ; or-

(3) Ripe herring sperm (protamine nucleate).

Glucose is added to keep the $p \mathrm{H}$ in the narrow limits within which the polar or nucleo-protein bodies develop.

Detoxication occurs by the precipitation in the medium of the endotoxin by the free nucleic acid, histone and protamines formed bio-chemically during culture.

Gonococcus nucleo-proteins are washed from the culture with 2 per cent. salt, leaving the endotoxin precipitated in the substance of the media.

These precipitations only occur at certain $\mathrm{H}$ ion concentrations and necessitates keeping the media at a constant $p \mathrm{H}$ during twenty hours of culture. 


\section{BRITISH JOURNAL OF VENEREAL DISEASES}

No. 3 Medium for the Preparation of Endotoxin

This is an acid autolysate of ox heart, which is buffered by suitable amounts of sodium bicarbonate, the reaction being $p \mathrm{H} 8.4$. An acid autolysate must be used in order to prevent the formation of gonococcus sclero-protein which is inert and developed at the expense of endotoxin. The successful preparation of the gonococcus endotoxin depends upon completely, by means of the organism's own enzymes, changing the 90 to 95 per cent. gonococcus phospho-protein through the stages of alkali meta-protein primary albumoses into deutero albumoses.

It is grown for ten days in an atmosphere of $\mathrm{CO}_{2}$ of appropriate percentage to keep the $p H$ constant at $7 \cdot 2$, then washed off with distilled water, and by means of repeated freezings and thawings, the whole of the albumoses are liberated from the bodies of the organism.

It is titred to I c.c. $=250$ millions and put up in colloidal silver.

In conclusion, I apologise for the discursive nature of this report and for its incompleteness.

I plead guilty to having pushed the manufacturing side of this research at the expense of the research itself and confirmatory tests, but the general sustained improvement on the clinical side has been my excuse, and, I hope, my justification. 